\title{
PRIME LAB SAMPLE HANDLING AND DATA ANALYSIS FOR ACCELERATOR- BASED BIOMEDICAL RADIOCARBON ANALYSIS
}

\author{
Darren J Hillegonds ${ }^{1}$ Rae Record ${ }^{2} \bullet$ Frank A Rickey $^{3}$ Steve Badylak ${ }^{2}$ George S Jackson ${ }^{3} \bullet$ \\ Abby Simmons-Byrd ${ }^{2} \cdot$ David Elmore $^{3}$ Michael E Lipschutz $^{1}$
}

\begin{abstract}
Processing and measurement of 200 biomedical samples has provided an opportunity to better understand the characteristics of accelerator mass spectrometry (AMS) analysis of such samples. We have utilized established procedures (Vogel 1992) and developed new methods for handling various biological samples. We have included secondary standards of known isotope ratio for all assays. A method of determining maximum precision for each unknown sample value is also reported. The presented data are an update of the ongoing radiocarbon AMS biomedical program at Purdue University.
\end{abstract}

\section{INTRODUCTION}

The utility of radioactive tracers to elucidate the behavior of xenobiotics within a biological system has been very important to medical science. Analysis of biomedical samples using accelerator mass spectrometry (AMS), with its high sensitivity and small sample requirements, is a developing field with the first concerted efforts occurring in 1988 (Vogel and Turteltaub 1992). Vanguard research has been conducted at Lawrence Livermore National Laboratory and the Center for Accelerator Mass Spectrometry (CAMS) in Livermore, California (Vogel et al. 1990; Turteltaub and Vogel 1996). A program of research after their lead was initiated at the Purdue Rare Isotope Measurement Laboratory (PRIME Lab) in 1996 (Rickey et al. 2000; Jackson et al. 2000). The present report is the first detailed description of methods developed for the study of radiocarbon biomedical samples at PRIME Lab. A separate report deals with the clinical results of this study (Record et al. 2001).

\section{METHODS}

\section{SIS Labeling and Implantation}

A study designed to determine the in-vivo fate of a surgical implant composed of small intestinal submucosa (SIS) has been previously described (Rickey et al. 2000). This study involves replacement of $35 \%$ to $45 \%$ of the urinary bladder in a dog model with radiocarbon-labeled SIS. This biomaterial, derived from porcine small intestine, is rapidly degraded in-vivo and facilitates the repair of organs and other tissues in humans and animals. SIS labeling was accomplished by administering weekly $10 \mu \mathrm{Ci}$ injections of ${ }^{14} \mathrm{C}$-proline to weanling piglets until they reached market weight, causing the entire animal to become enriched in ${ }^{14} \mathrm{C}$. SIS consists of $90 \%$ collagen by dry weight, and because of the relatively high concentration of proline in tissue collagen, the harvested SIS was 3600 \pm 300 times modern ${ }^{14} \mathrm{C}$ activity.

The labeled SIS was used to manufacture devices suitable for the repair of urinary bladder defects in dogs. After implantation, each dog was sacrificed at time points from three days to one year, whereupon samples of various organs were removed and stored for AMS analysis. Blood, urine, and feces samples were taken at regular intervals, from before implantation until the date of sacrifice, to measure potential routes of excretion of the labeled material as the SIS device was degraded in-vivo.

\footnotetext{
${ }^{1}$ Purdue University, Department of Chemistry, 1393 Brown Building, West Lafayette, Indiana, 47907, USA.

Email: terminus_post_quem@yahoo.com orderwood@purdue.edu.

${ }^{2}$ Purdue University, Department of Biomedical Engineering, 1296 Potter Center, West Lafayette, Indiana, 47907, USA

${ }^{3}$ PRIME Lab, Purdue University, Department of Physics, 1396 Physics Building, West Lafayette, Indiana, 47907, USA
}

(C) 2001 by the Arizona Board of Regents on behalf of the University of Arizona RADIOCARBON, Vol 43, Nr 2A, 2001, p 305-311

Proceedings of the 17 th International ${ }^{14} \mathrm{C}$ Conference, edited by I Carmi and E Boaretto 


\section{Initial Screening with LSC}

To avoid contamination of the AMS processing laboratory, all samples were screened with a Packard Tri-Carb ${ }^{\circledR}$ 1600CA liquid scintillation counter (LSC). For a well-understood study, this screening would not be necessary, but we felt it important to have such a method in place, should it be needed for future projects. Small pieces of tissue $(\sim 100 \mathrm{mg})$ were placed into scintillation vials and dissolved in Soluene ${ }^{\circledR}-350$ (Packard Instruments, Meriden, CT) at $50{ }^{\circ} \mathrm{C}$ for $2-4 \mathrm{hr}$. Feces $(\sim 20 \mathrm{mg})$ were placed into scintillation vials and water added for rehydration, followed by addition of Soluene ${ }^{\circledR}-350$. After $1-2 \mathrm{hr}$ at $50{ }^{\circ} \mathrm{C}$, isopropanol was added and the feces were incubated at $50{ }^{\circ} \mathrm{C}$ for 2 additional hr, after which $\mathrm{H}_{2} \mathrm{O}_{2}$ was added. Blood $(100 \mu \mathrm{L})$ was placed in scintillation vials, a mixture of Soluene ${ }^{\circledR}-350$ and isopropanol was added, and the mixture was incubated at $50{ }^{\circ} \mathrm{C}$ for $2 \mathrm{hr}$. $\mathrm{H}_{2} \mathrm{O}_{2}$ was added and the samples incubated at $50{ }^{\circ} \mathrm{C}$ for another 30 minutes. Urine $(200 \mu \mathrm{L})$ was placed in scintillation vials and Solune ${ }^{\circledR}-350$ was added. Prior to counting, scintillation fluid (Hionic-Fluor $^{\mathrm{TM}}$, Packard Instruments, Meriden, CT) was added to all samples. The LSC had a relatively high background ( $\sim 30 \mathrm{dpm})$, and its utility was limited to screening for hot samples and qualitative determinations thereof.

\section{Sampling for AMS Analysis}

Tissue samples to be measured on the accelerator were initially freeze-dried in a process more commonly known as lyophilization. Organ samples $(20 \mathrm{mg})$ were placed into the bottom of $6 \times 60 \mathrm{~mm}$ quartz test tubes. Four or five of these tubes were placed into a plastic $50 \mathrm{~mL}$ centrifuge tube, each with a $8 \times 75 \mathrm{~mm}$ glass culture tube covering the open end to reduce the probability of cross contamination. The cap of the centrifuge tube was vented, and several of these setups were lyophilized for $24 \mathrm{hr}$. Feces, blood, and urine were treated equivalently, with sample amounts of $50 \mathrm{mg}, 50 \mu \mathrm{l}$, and $100 \mu \mathrm{L}$ respectively.

Combustion and reduction procedures follow those developed at CAMS (Vogel 1992), with a few minor differences. The quartz tubes containing dried samples were placed directly into $9 \times 1500 \mathrm{~mm}$ quartz tubes, thus eliminating any sample transfer steps which could lead to contamination. Each $9 \mathrm{~mm}$ tube had a sealed breakable tip on one end, and contained $0.5 \mathrm{~g} \mathrm{CuO}$ plus a small $(2 \times 5 \mathrm{~mm})$ piece of silver foil. Standards and blanks were prepared simultaneously, with portions of each weighed into $6 \times 60 \mathrm{~mm}$ quartz tubes. The combustion tubes were marked on the outside with a heatresistant marker (CEM, Mathews, NC), evacuated, flame-sealed, and heated in a furnace to $900{ }^{\circ} \mathrm{C}$ for three hours. The position in the furnace was recorded and each tube was physically separated from the others so an explosion of one would not destroy additional combustion tubes.

After heating, combustion tubes were attached to a disposable plastic manifold to which a reduction tube was also attached (cf. Vogel 1992). Reduction tubes contained about $3 \mathrm{mg}$ iron (as opposed to the cobalt used by Vogel 1992), $200 \mathrm{mg}$ 20-mesh zinc, and $40 \mathrm{mg} \mathrm{TiH}_{2}$. Because our ion source accommodates eight samples at a time (in general one blank, two standards and five unknowns), we sequentially prepared samples on an eight-position vacuum line. Each combustion tube was cooled in dry ice/ethanol to remove water and the tubing above the disposable manifold sealed with a pinch clamp. The bottom of the reduction tube was cooled in liquid nitrogen and the tip of the combustion tube broken, allowing the $\mathrm{CO}_{2}$ transfer. After 5 minutes, the pinch clamp was removed and incondensable gasses pumped away. The reduction tube was then flame-sealed, removed from the manifold, and heated to $500^{\circ} \mathrm{C}$ for $3 \mathrm{hr}$, then at $550{ }^{\circ} \mathrm{C}$ for $2 \mathrm{hr}$. As with the combustion, reduction tubes were heated in batches with each sample in an individual steel or ceramic tube. 
The heated reduction tubes were broken and the graphite/iron mixtures pressed into copper holders. AMS analysis followed, with each unknown and blank normalized using the two concurrently processed standards.

\section{RESULTS AND DISCUSSION}

\section{Reduction Failure}

Approximately $15 \%$ of the 150 unknown samples included in this study failed to yield ion currents necessary for reliable ${ }^{14} \mathrm{C} /{ }^{12} \mathrm{C}$ determination. The reasons for these failures were not completely understood, as there does not seem to be any correlation with sample size and all samples were treated equivalently. Perhaps the reduction conditions were not optimal, and different quantities of reagents might reduce the failure rate (cf. Vogel 1992). Because samples were small relative to material removed from the test subjects, failures were ignored and a replacement sample was made. For research where such a failure rate is not acceptable, a more robust method of reduction (or better understanding of this one) would be necessary. Because the present method allows large numbers of samples to be prepared in shorter times (and more inexpensively) than more typical reduction schemes (Jull et al. 1986; Wilson 1992), we feel the benefits outweigh the drawbacks.

\section{Data Handling Procedures}

\section{Initial Screening for Outliers}

After all samples have been run, individual measurements were checked for outliers. The ${ }^{14} \mathrm{C}$ to ${ }^{13} \mathrm{C}$ ratio was measured at least 12 times, separated into three so-called sequences. If the weighted average of each sequence deviated by more than $3 \sigma$ from one of the measurements in that sequence, the outlier was discarded. Such deletions were uncommon ( $<1 \%$ of the data) and generally prompted by a significant ion current change during measurement.

\section{Assigning Uncertainties}

All AMS data were normalized to standards of known isotopic ratio. We used two standards for every five unknowns to correct for drift in machine parameters during the relatively long measurement times needed for slow magnet switching. To compensate for analysis variables, all blank and unknown sequence mean values were divided by the average normalization factor of the standard sequences run immediately prior to and after them. This correction factor was computed by dividing the calculated ${ }^{14} \mathrm{C} /{ }^{12} \mathrm{C}$ for each standard sequence by the known ratio of $1.618 \times 10^{-12}$ (PRIME Lab uses ${ }^{14} \mathrm{C}$ standard SRM 4990C).

To better understand uncertainties in measured ${ }^{14} \mathrm{C} /{ }^{13} \mathrm{C}$, we examined the scatter in isotope ratio and ${ }^{13} \mathrm{C}^{3+}$ ion current for SRM 4990C samples. The January and May 2000 runs had high correlations between ${ }^{13} \mathrm{C}^{3+}$ ion current and normalization factor $(\mathrm{p}<0.001)$, resulting in a $15 \%$ difference in normalization factor over an order of magnitude variation in ion current. Since we do not understand the origin of this dependence, we did not correct data for it but rather increased the uncertainty of results for samples with beam currents different from those of the standards. The reasons for these ion current deviations are being investigated. Differing target characteristics could arise from various minor contaminants fouling the reduction, as well as sample size differences, leading to production of different forms of graphite and varying graphite/iron ratios. 


\section{Secondary Standard Results}

Results for samples of SRM 4990C processed and measured as unknowns are shown in Table 1. The overall accuracy is somewhat lower than statistical expectations $(n=27)$, with data points outside one sigma being excessively so: only $81 \%$ fall within $2 \sigma$ and $93 \%$ within $3 \sigma$ of the true value, indicating some unaccounted-for source of error or non-Gaussian behavior. These secondary standards have weighted and unweighted means very close to the expected value $(+0.06 \%$ and $+0.3 \%$ respectively), showing that our methods of combustion, reduction, and AMS analysis yield accurate data. It should be noted that from the standpoint of accuracy, use of weighted averages is strongly preferred, as it tends to decrease detrimental effects of outliers: such deviant values are generally less precise, both in unknown measurements and in normalization.

Table 1 Fraction modern for secondary standards from the August 1999, January 2000, and May 2000 runs. Samples are SRM 4990C processed and run as unknowns, normalized to $\delta^{13} \mathrm{C}$ of $-25 \%$, the accepted value is 1.3506 .

\begin{tabular}{lcccccc}
\hline & & & \multicolumn{4}{c}{ Individual values within } \\
\cline { 4 - 7 } Run & Unweighted mean & Weighted mean & $1 \sigma$ & $2 \sigma$ & $3 \sigma$ & $4 \sigma$ \\
\hline August 1999 $(\mathrm{n}=6)$ & $1.39 \pm 0.02(1 \%)$ & $1.36 \pm 0.01(0.8 \%)$ & 100 & 100 & 100 & 100 \\
January 2000 $(\mathrm{n}=8)$ & $1.36 \pm 0.04(3 \%)$ & $1.353 \pm 0.009(0.7 \%)$ & 63 & 63 & 88 & 100 \\
May 2000 $(\mathrm{n}=13)$ & $1.35 \pm 0.03(2 \%)$ & $1.350 \pm 0.004(0.3 \%)$ & 69 & 85 & 92 & 100 \\
All runs $(\mathrm{n}=27)$ & $1.36 \pm 0.03(2 \%)$ & $1.351 \pm 0.003(0.2 \%)$ & 74 & 81 & 93 & 100 \\
\hline
\end{tabular}

\section{Replicate Analyses}

\section{Pre-operative Samples}

Samples collected prior to implantation of the labeled SIS (i.e. pre-operative samples) are a good indicator of the reproducibility of the methods employed because they should all have contemporary ${ }^{14} \mathrm{C}$ levels. The pre-operative samples collected consisted of bladder resected from each dog and samples of blood, urine and feces collected before surgery: Tables 2-5 show the AMS results for these samples, presented in units of fraction modern. The most clinically relevant data lie in the elevation of ${ }^{14} \mathrm{C}$ over pre-operative levels, so future work should be reported in reference to pre-operative ${ }^{14} \mathrm{C}$ content.

Measurements of pre-operative blood (Table 2) and bladder (Table 3) samples have variabilities of $3.4 \%$ and $6.8 \%$, respectively. The inconsistent ${ }^{14} \mathrm{C}$ content of the 1 -week dog pre-operative bladder is reflected in the urine data (Table 4) and we believe this indicates a potential pre-analysis contamination: data from the 1-week dog is not included in the average values reported in Tables 2 and 3 .

Data collected subsequent to submission of this paper helps explain the pre-operative urine data, but are not individually reported because they were not in the reviewed paper. Replicate analysis of PL001144A (pre-operative urine from the dog sacrificed 1 week after implantation) proved consistent with PL001187A and PL001129A; PL001144A is likely the result of some transient analysis error and we consider it an outlier. The high ${ }^{14} \mathrm{C}$ content of the PL001158A was confirmed in reanalysis, prompting us to eliminate the 1 week dog from consideration. The weighted average pre-operative urine value reported in Table 4 includes only PL001187A and PL001129A; inclusion of five additional pre-operative urine analyses yielded a revised average fraction modern of $1.12 \pm 0.05$. Three additional pre-operative bladder samples were also measured, giving a revised average fraction modern of $1.22 \pm 0.09$. 
Table 2 Pre-operative blood samples from dogs sacrificed at the time specified past implantation. The 1-week dog is not included in the average due to high variability in urine and bladder data.

\begin{tabular}{lllc}
\hline Dog & Lab ID & $\mathrm{F}_{\mathrm{m}}$ & $1 \sigma$ error \\
\hline 3 day & PL001095A & 1.24 & 0.01 \\
1 week & PL001152A & 1.20 & 0.01 \\
8 week & average value $^{\mathrm{a}}$ & 1.166 & 0.007 \\
12 week & average value $^{\mathrm{b}}$ & 1.152 & 0.007 \\
16 week & PL001166A & 1.14 & 0.02 \\
24 week & PL001135A & 1.16 & 0.02 \\
36 week & PL001172A & 1.14 & 0.02 \\
12 month & PL001147A & 1.17 & 0.02 \\
\hline & Weighted mean: & 1.17 & \\
& Standard deviation: & 0.04 & \\
& \% variation: & 3.4 & \\
\hline
\end{tabular}

aPL001178A and PL001177A

${ }^{\mathrm{b} P L 001122}$ and PL001123A

Table 3 Pre-operative bladder samples from dogs sacrificed at the time specified past implantation. Weighted average and standard deviation do not include the one-week dog (see text).

\begin{tabular}{lllc}
\hline Dog & Lab identification & $\mathrm{F}_{\mathrm{m}}$ & $1 \sigma$ error \\
\hline 1 week & PL991742A & 1.08 & 0.06 \\
1 week & PL000102A & 1.6 & 0.1 \\
2 week & PL991743A & 1.04 & 0.05 \\
2 week & PL000103A & 1.19 & 0.09 \\
8 week & PL000109A & 1.27 & 0.04 \\
12 week & PL000110A & 1.16 & 0.04 \\
16 week & PL001160A & 1.18 & 0.01 \\
\hline & Weighted mean: & 1.17 & \\
& Standard deviation: & 0.08 & \\
& \% variation: & 6.8 & \\
\hline
\end{tabular}

Pre-operative feces samples (Table 5) are lower in ${ }^{14} \mathrm{C}$ than pre-operative blood and bladder, and are more consistent with the revised urine data. The higher activity of the bladder samples could be indicative of the integrated lifetime ${ }^{14} \mathrm{C}$ dose, which would incorporate more bomb pulse ${ }^{14} \mathrm{C}$ into conserved tissues than excreta. The pre-operative feces and urine should be more consistent with the contemporary ${ }^{14} \mathrm{C}$ levels in the dog's food, and therefore lower in ${ }^{14} \mathrm{C}$ than body organs. Blood contains a significant portion of circulating proteins, and should also reflect integration of more bomb pulse carbon; this is also indicated by our results. 
Table 4 Pre-operative urine samples from dogs sacrificed at the time specified past implantation. Weighted average and standard deviation do not include the oneweek or two-week results (see text).

\begin{tabular}{lllc}
\hline Dog & Lab identification & $\mathrm{F}_{\mathrm{m}}$ & $1 \sigma$ error \\
\hline 1 week & PL001158A & 1.88 & 0.06 \\
2 week & PL001144A & 6.19 & 0.05 \\
8 week & PL001187A & 1.06 & 0.02 \\
12 week & PL001129A & 1.09 & 0.02 \\
\hline & Weighted mean: & 1.07 & \\
& Standard deviation: & 0.02 & \\
& \% variation: & 1.9 & \\
\hline
\end{tabular}

Table 5 Pre-operative feces samples from dogs sacrificed at the time specified past implantation

\begin{tabular}{lllc}
\hline Dog & Lab identification & $\mathrm{F}_{\mathrm{m}}$ & $1 \sigma$ error \\
\hline 1 week & PL991744A & 1.13 & 0.05 \\
2 week & PL000099A & 1.14 & 0.03 \\
4 week & PL000090A & 1.108 & 0.009 \\
\hline & Weighted mean: & 1.11 & \\
& Standard deviation: & 0.02 & \\
& \% variation: & 1.8 & \\
\hline
\end{tabular}

Table 6 Dog bladder samples used to determine the potential for cross contamination in the lyophilization step. Samples were taken from an animal not involved in the present study.

\begin{tabular}{llc}
\hline Lab identification & $\mathrm{F}_{\mathrm{m}}$ & $1 \sigma$ error \\
\hline PL001100A & 1.126 & 0.009 \\
PL001103A & 1.109 & 0.009 \\
PL001104A & 1.115 & 0.009 \\
PL001106A & 1.10 & 0.01 \\
PL001107A & 1.13 & 0.01 \\
PL001108A & 1.23 & 0.01 \\
PL001109A & 1.12 & 0.01 \\
PL001101A & 1.13 & 0.01 \\
\hline Weighted mean: & 1.13 & \\
Standard deviation: & 0.04 & \\
\% variation: & 3.5 & \\
\hline
\end{tabular}

\section{Bladder Controls to Test for Crosstalk}

To test for contamination in the lyophilization step, we processed a set of control samples along with high ${ }^{14} \mathrm{C}$-activity samples, these results are presented in Table 6 . One of the data points is higher than the others, but the overall $1 \sigma$ variation is only $3.6 \%$. Because the controls 
were dried with high activity samples $\left({ }^{14} \mathrm{C} /{ }^{12} \mathrm{C} \sim 10^{3}\right.$ higher than the controls), any contamination would be worse than for all other unknowns in this study: the results show no indication for this. These data also illustrate that significant differences among unknowns should differ by not less than $3.6 \%$. Because this value is about twice the variation of the unweighted mean values for quality control samples shown in Table 1, it is apparent that the major source of scatter in the data is not from the AMS analysis, but from biological diversity in the research subjects.

\section{CONCLUSIONS}

We have demonstrated a successful start in the development of ${ }^{14} \mathrm{C}$ biomedical sample processing at PRIME Lab. Combustion and reduction procedures reproduce secondary standard values, notwithstanding an occasional reduction failure. Replicate analyses of biological samples show good reproducibility, but some intermittent contamination persists. We anticipate that planned improvements to the AMS and more experience in chemical preparation will allow higher precision and increased throughput.

\section{ACKNOWLEDGMENTS}

We gratefully acknowledge the tireless work of Tom Miller (PRIME Lab). We also thank the reviewer for insightful comments leading to significant improvements in the quality of this publication. This work was supported by Purdue University re-investment funds.

\section{REFERENCES}

Jackson GS, Elmore D, Rickey FA, Musameh SA, Sharma P, Hillegonds D, Coury L, Kissinger P. 2000. The PRIME Lab biomedical program. Nuclear Instruments and Methods in Physics Research B172:899903.

Jull AJT, Donahue DJ, Hatheway AL, Linick, TW, Toolin, LJ. 1986. Production of graphite targets by deposition from $\mathrm{CO} / \mathrm{H}_{2}$ for precision accelerator ${ }^{14} \mathrm{C}$ measurements. Radiocarbon 28(2A):191-7.

Record RD, Hillegonds DJ, Simmons C, Tullius R, Rickey FA, Elmore D, Badylak SF. 2001. In vivo degradation of ${ }^{14} \mathrm{C}$-labeled small intestinal submucosa (SIS) when used for urinary bladder repair. Biomate rials 22:2653-9.

Rickey FA, Elmore D, Hillegonds D, Badylak S, Record R, Simmons-Byrd A. 2000. Regeneration of tissue about an animal-based scaffold: AMS studies of the fate of the scaffold. Nuclear Instruments and Methods in Physics Research B172:904-9.
Turteltaub KW, Vogel JS. 1996. Applications of accelerator mass spectrometry in toxicology: a highly sensitive tool for low-level isotope measurements. In: Burlingame AL, Carr SA, editors. Mass Spectrometry in the Biological Sciences. New Jersey: Humana Press.

Vogel JS, Turteltaub KW. 1992. Biomolecular tracing through accelerator mass spectrometry. Trends in Analytical Chemistry 11(4):142-9.

Vogel JS, Turteltaub KW, Felton JS, Gledhill BL, Nelson DE, Southon JR, Proctor ID, Davis JC. 1990. Application of AMS to the biomedical sciences. Nuclear Instruments and Methods in Physics Research B52:52430.

Vogel JS. 1992. Rapid production of graphite without contamination for biomedical AMS. 1992. Radiocarbon 34(3):344-50.

Wilson AT. 1992. A simple technique for converting $\mathrm{CO}_{2}$ to AMS graphite. 1992. Radiocarbon 34(3):318-20. 